Research Article

\title{
Automatic Liquid Filling Machine Using Flow Sensor and Arduino Pro Mini
}

\author{
Rahmad Hidayat ${ }^{*}$, Handayani Saptaji Winahyu ${ }^{2}$ \\ 1 Department of Electrical Engineering, Sekolah Tinggi Teknologi Mandala, 16911, Indonesia \\ 2 Postgraduate Student, Polytechnic State of Semarang 50275, Indonesia
}

Article history:

Submission January 2020

Revised February 2020

Accepted February 2020

*Corresponding author:

E-mail: rhidayat4000@gmail.com

\begin{abstract}
The Small and Medium Enterprise (SME), which is especially involved in the liquid filling industry (such as mineral water, tea, liquid milk, juice, etc.), needs some device that has the ability to measure liquid automatically and accurately into some container. Based on the 'Water Fall' method, this study carries out a procedure for making compact and efficient tools ('requirements definition' stage), using a keypad start button, flow sensor, temperature sensor, fan, buzzer, LCD 1602, relay driver ('system \& software design' stage), implement a schematic drawing of the circuit and install all hardware inside the casing ('implementation and unit testing' stage), install accessories, set parameters and volume target ('integration and system testing ' stage) and re-calibrate the device ('operation and maintenance' stage). After the measurement experiment, we got that Fillco (the name of the device) has a bigger error for measurement below $1000 \mathrm{ml} \mathrm{(0.6-15 \% )}$ and better accuracy measurement for targeted volume upper than $1000 \mathrm{ml}$ (0.04-0.20\%). Fillco also able to memorize and reset liquid volume.
\end{abstract}

Keywords: Liquid filling, automatic filling machine, flow sensor, Arduino pro mini, solenoid valve

\section{Introduction}

In the small industries activities, especially for liquid filling industry (such as mineral water, tea, liquid milk, juice, etc.), often to refill liquids into containers manually. As a result, the liquid that is filled in the containers is inaccurate so it can cause losses both in terms of cost and time. Because the liquid filling activity in the container is carried out continuously, as an accumulated losses result become greater so it will reduce industry profits significantly and make the Small and Medium Enterprise (SME) does not grow in the next future. Thus, we need a device that able to measure the volume of liquid effectively, efficiently and at affordable prices. Related with small scale industry players who have not been technology literate, device installation and operation must be easy and practical and does not require a large space so it will be easily installed into owner business place.

\section{State of the art}

Today, the world is moving towards product automation to increase the quality and quantity to meet customer demand. One industrial automation in the field of bottle filling involves the use of cylinders, pneumatic systems, and PLCs, but these systems operate inexpensive ways. Although all of this is available on the market, there are still small industries that use manual methods to fill bottles. The obstacle for them not to use automation technology is the high cost. This study focuses on making a system to measure the volume of liquid-filled in bottles using Arduino and building a prototype of an inexpensive filling machine. Arduino microcontrollers are cheap and very reliable to do 
the task. Before filling the machine using cylinders and pneumatics, workers need to fill bottles manually using taps and tanks. There are still spills of water, the amount of water is not the same, slow in production, and cleanliness problems can cause harm to the company. Automatic filling machines will reduce this loss and reduce labor costs and eliminate other problems faced by small industries [1] [2] [3].

In [4], an Arduino-based automatic bottle filling and capping system were made. The expensive PLC boards used in the large-scale bottle filling industry were replaced by Arduino UNO boards which were much cheaper than PLCs. Arduino is used to controlling the whole system with Arduino coding. This system uses IR sensors, relay switches, solenoid valves, dc motors, and plungers. The result is that inexpensive machine installation costs and less time-consuming work compared to manual bottle filling techniques.

Meanwhile, the water volume measurement system using an ultrasonic sensor was developed to replace the cylindrical piston where the automatic filling machine available on the market is complicated and difficult to clean because of the installation of a piston requires a pneumatic system to operate. An additional tank called a measuring tank with an ultrasonic sensor is installed to measure the volume of water as desired before filling in the bottle. Two solenoid valves to control the flow of water between the storage tank to the measurement tank and the measurement tank to the nozzle. A measuring tank with an ultrasonic sensor is placed on the tank cap to measure the amount of water that is filled in it. The amount of water is measured by weight to get better results. The desired amount of water is set to 200 grams and then uses the same ultrasonic sensor to measure distances. From the experimental results, the percentage of errors is quite large at $4.9 \%$ due to the fast flow of water and the surface of the water is still moving (not stagnant) [5].

In order for the production process will run smoothly, the amount of labor and production time will be reduced and costs will be lower, [6] developing a chemical liquid dispenser machine for the pharmaceutical industry. Their system is controlled by the AT89C52 microcontroller to improve the quality of the dispenser machine's volume measurement. The same system was designed to make temperature and volume mixing machines using a microcontroller [7]. Its main function is to mix various liquids that require certain rations and temperatures. AT89S51 microcontroller and two LM35 temperature sensors were used to develop this system.

\section{Methods}

The method applied in this research is 'Water Fall method' which includes several stages:

\section{a. Requirements Definition}

Based on the needs in the field, this research is focused on making device/tools that meet the following requirements:

- Able to calculate liquid volume accurately

- Small dimensions, not require large space for installation

- Easy installation

- Easy operation

- Long durability (to support fast BEP for SME)

\section{b. System and software design}

Arduino Pro Mini is used for a control system, because of its compact physical form factor and its same specifications as Arduino Uno. The dimensions of this controller greatly help smaller casing designs so it will be easily installed anywhere [8]. For input terminal from an automatic liquid filling device, consisting of:

- Keypad, to enter data settings

- Start button, to start the liquid filling measurement process

- Flow sensor, to calculate liquid flow rate and volume.

- Temperature sensor, to detect overheat in the device

Whereas for the output port, it consists of:

- Exhaust fan, to prevent the device from overheating.

- Buzzer, as a process indicator, to start or stop volume measurement 
- LCD 1602, to display volume measurement.

- Relay driver for solenoid valve (electronic faucets opening/closing).

- Relay driver for an electric pump.

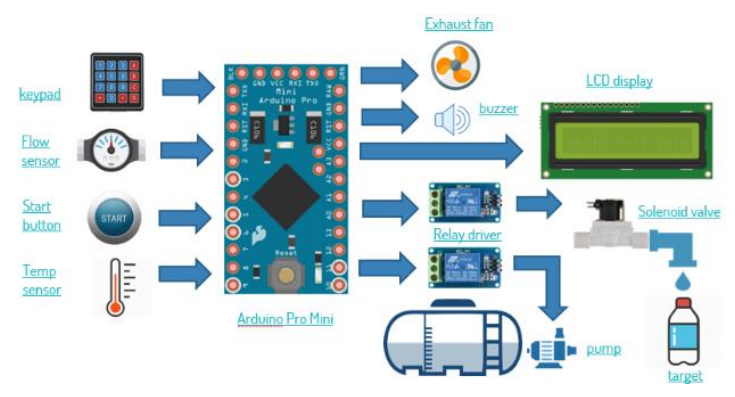

Figure 1. Hardware design

For the software design, shown in the flowchart image.

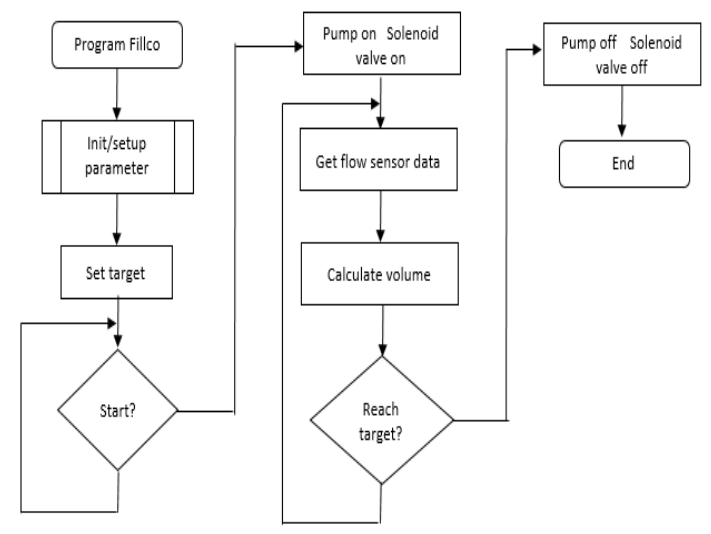

Figure 2. Flowchart program

\section{c. Implementation and Unit Testing}

The hardware and software design above is then implemented as an electronic circuit.

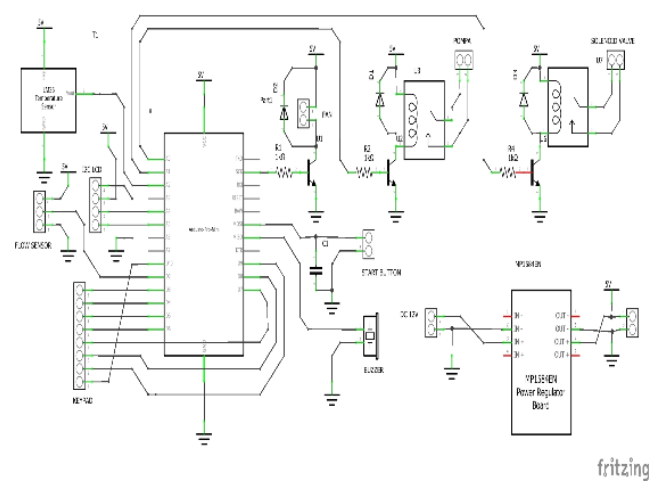

Figure 3. Schematic drawing
All components are then assembled and put into a casing and labeled as shown in Figure 4.

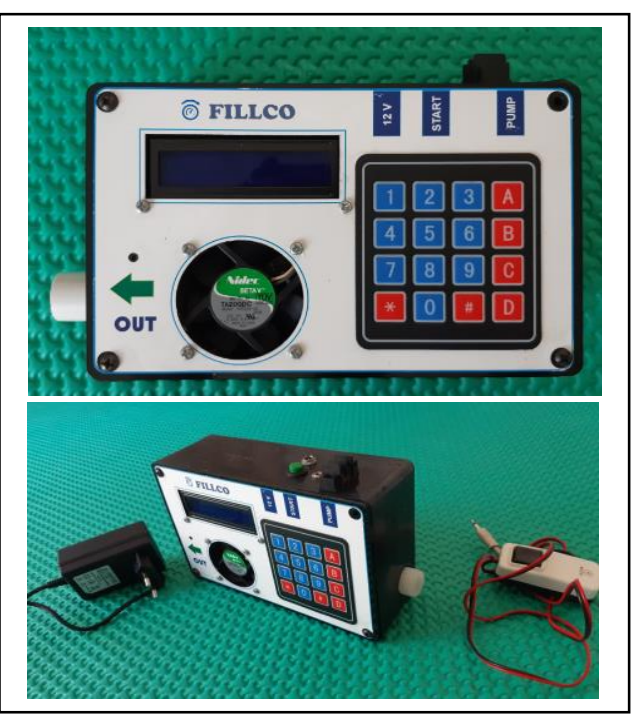

Figure 4. The device after assembling

\section{d. Integration and system testing}

To integrate and test the system, the following procedure must be conducted:

- Wire all accessories (power supply, button, pump cable) into terminals.

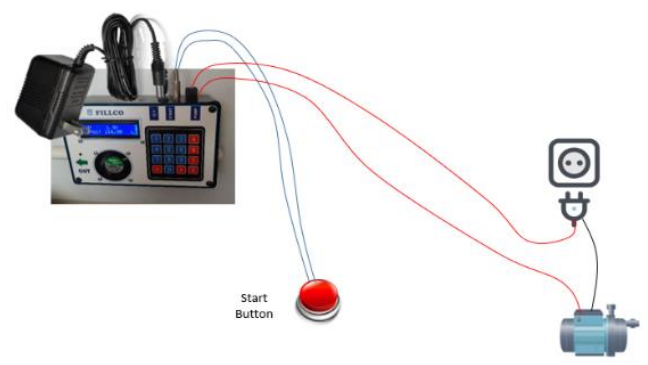

Figure 5. Wiring diagram

- The setting device from the keypad with these rules (4 times keystroke in sequence to enter menu).

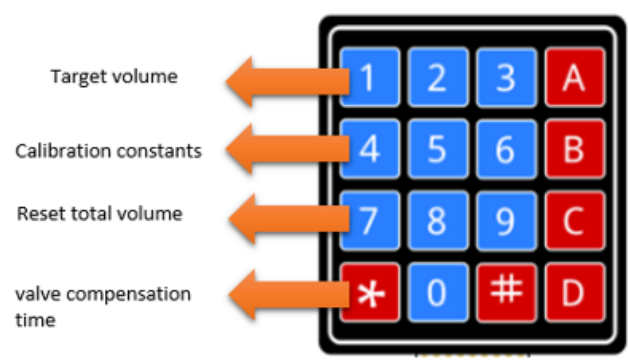

Figure 6. Setting parameters 
- Set a targeted volume, then press the 'Start Button' to begin the process

e. Operation and Maintenance

- The device need to recalibrate if the pump is changed.

- If water debit less than $7 \mathrm{~L} /$ minute please recalibrate.

- Make sure your pump is in proper condition, test on/off manually before joined into Fillco terminal.

- Recommended temperature room is $25^{\circ} \mathrm{C}$

\section{Results and Discussion}

From the implementation of the automatic liquid filling device design above, volume measurement results are obtained as followCalculation of relative errors based on the formula:

Error relatif $(\%)=$ (modulo error/measured volume) x $100 \%$

Table 1. Liquid volume measurement results

\begin{tabular}{ccccc}
\hline No & $\begin{array}{c}\text { Target } \\
\text { Vol. } \\
(\mathrm{mL})\end{array}$ & $\begin{array}{c}\text { Measured } \\
\text { Vol. } \\
(\mathrm{mL})\end{array}$ & $\begin{array}{c}\text { Mod } \\
\text { error }\end{array}$ & $\begin{array}{c}\text { \% er- } \\
\text { ror } \\
\text { rel. }\end{array}$ \\
\hline 1 & 100 & 110 & 10 & 10.00 \\
\hline 2 & 100 & 105 & 5 & 5.00 \\
\hline 3 & 100 & 115 & 15 & 15.00 \\
\hline 4 & 100 & 103 & 3 & 3.00 \\
\hline 5 & 100 & 112 & 12 & 12.00 \\
\hline 6 & 500 & 505 & 5 & 1.00 \\
\hline 7 & 500 & 507 & 7 & 1.40 \\
\hline 8 & 500 & 502 & 2 & 0.40 \\
\hline 9 & 500 & 510 & 10 & 2.00 \\
\hline 10 & 500 & 503 & 3 & 0.60 \\
\hline 11 & 1000 & 1000 & 0 & 0.00 \\
\hline 12 & 1000 & 1001 & 1 & 0.10 \\
\hline 13 & 1000 & 1002 & 2 & 0.20 \\
\hline 14 & 1000 & 1000 & 0 & 0.00 \\
\hline 15 & 1000 & 1001 & 1 & 0.10 \\
\hline 16 & 1500 & 1501 & 1 & 0.07 \\
\hline 17 & 1500 & 1500 & 0 & 0.00 \\
\hline 18 & 1500 & 1502 & 2 & 0.13 \\
\hline 19 & 1500 & 1501 & 1 & 0.07 \\
\hline 20 & 1500 & 1500 & 0 & 0.00 \\
\hline & & & & \\
\hline
\end{tabular}

\begin{tabular}{lllll}
\hline 21 & 2000 & 2000 & 0 & 0.00 \\
\hline 22 & 2000 & 2002 & 2 & 0.10 \\
\hline 23 & 2000 & 2000 & 0 & 0.00 \\
\hline 24 & 2000 & 2001 & 1 & 0.05 \\
\hline 25 & 2000 & 2000 & 0 & 0.00 \\
\hline 26 & 2500 & 2503 & 3 & 0.12 \\
\hline 27 & 2500 & 2500 & 0 & 0.00 \\
\hline 28 & 2500 & 2502 & 2 & 0.08 \\
\hline 29 & 2500 & 2500 & 0 & 0.00 \\
\hline 30 & 2500 & 2501 & 1 & 0.04 \\
\hline 31 & 3000 & 3003 & 3 & 0.10 \\
\hline 32 & 3000 & 3003 & 3 & 0.10 \\
\hline 33 & 3000 & 3002 & 2 & 0.07 \\
\hline 34 & 3000 & 3005 & 5 & 0.17 \\
\hline 35 & 3000 & 3000 & 0 & 0.00 \\
\hline
\end{tabular}

\section{Conclusion}

In this system, the flow sensor and Arduino Pro Mini have been implemented well. Fillco (name of the device above) able to measure liquid volume as targeted volume, set by the user. As data result, the liquid level of $1000 \mathrm{~mL}$ is the threshold of the measurement value between the larger error and the better-targeted error. The whole process is more reliable. Fillco able to memorize liquid volume (and reset too), so the user gets an advantage for this feature.

\section{Acknowledgment}

The authors say thanks to Polytechnic State of Semarang and Sekolah Tinggi Teknologi Mandala for supporting this research.

\section{References}

1. Bipin M, Pallavi K, Girish D (2015) Automated Bottle Filling engineering students [https://peer.asee.org/an-automatedbottle-filling-and-capping-project-for-freshman-engineering-students.pdf], accessed on November, $4^{\text {th }} 2019$.

3. Kalaiselvi, Kalaiselvi, Praveena, Aakanksha R, Dhanya S (2012) PLC based automatic bottle filling and capping system with user-defined volume selection. IJETAE 8: 134-137.

4. Lakshmeesha, Prajwal K, Shetty G, Sriganesh, Ravindran KN (2018) Automated bottle filling \& capping machine using Arduino. IJIRE 5:92-96. System. IRJET. 7: 771-776.

2. An automated bottle filling and capping project for freshman 
5. Muhammad S, Che G (2017) Volume measuring system using arduino for automatic liquid filling machine. IJAER 24: 14505-14509.

6. Rajesh GK, Chadrashekhar N S (2012) Design and fabrication of liquid dispensing machine using automatic control for engg. industry. IJITEE 5: 38-43.
7. Sastry, Srinivas, Krishna, Sesha KK (2010) An automated microcontroller based liquid mixing system. Interface. IJCSE. 8: 2648-2651.

8. Emily G (2012) Environmental Monitoring with Arduino. Sebastopol, USA, O’Reilly Media, Inc. 